\title{
Muséologies
}

Les cahiers d'études supérieures

\section{Dialogue avec Mme Louise Déry}

\section{Stéphanie Corriveau}

Volume 1, numéro 2, avril 2007

URI : https://id.erudit.org/iderudit/1033607ar

DOI : https://doi.org/10.7202/1033607ar

Aller au sommaire du numéro

Éditeur(s)

Association Québécoise de Promotion des Recherches Étudiantes en Muséologie (AQPREM)

ISSN

1718-5181 (imprimé) 1929-7815 (numérique)

Découvrir la revue

Citer ce document

Corriveau, S. (2007). Dialogue avec Mme Louise Déry. Muséologies, 1(2), 42-49. https://doi.org/10.7202/1033607ar

Ce document est protégé par la loi sur le droit d'auteur. L’utilisation des services d'Érudit (y compris la reproduction) est assujettie à sa politique d'utilisation que vous pouvez consulter en ligne.

https://apropos.erudit.org/fr/usagers/politique-dutilisation/
Cet article est diffusé et préservé par Érudit.

Érudit est un consortium interuniversitaire sans but lucratif composé de l’Université de Montréal, l’Université Laval et l'Université du Québec à Montréal. Il a pour mission la promotion et la valorisation de la recherche. https://www.erudit.org/fr/ 


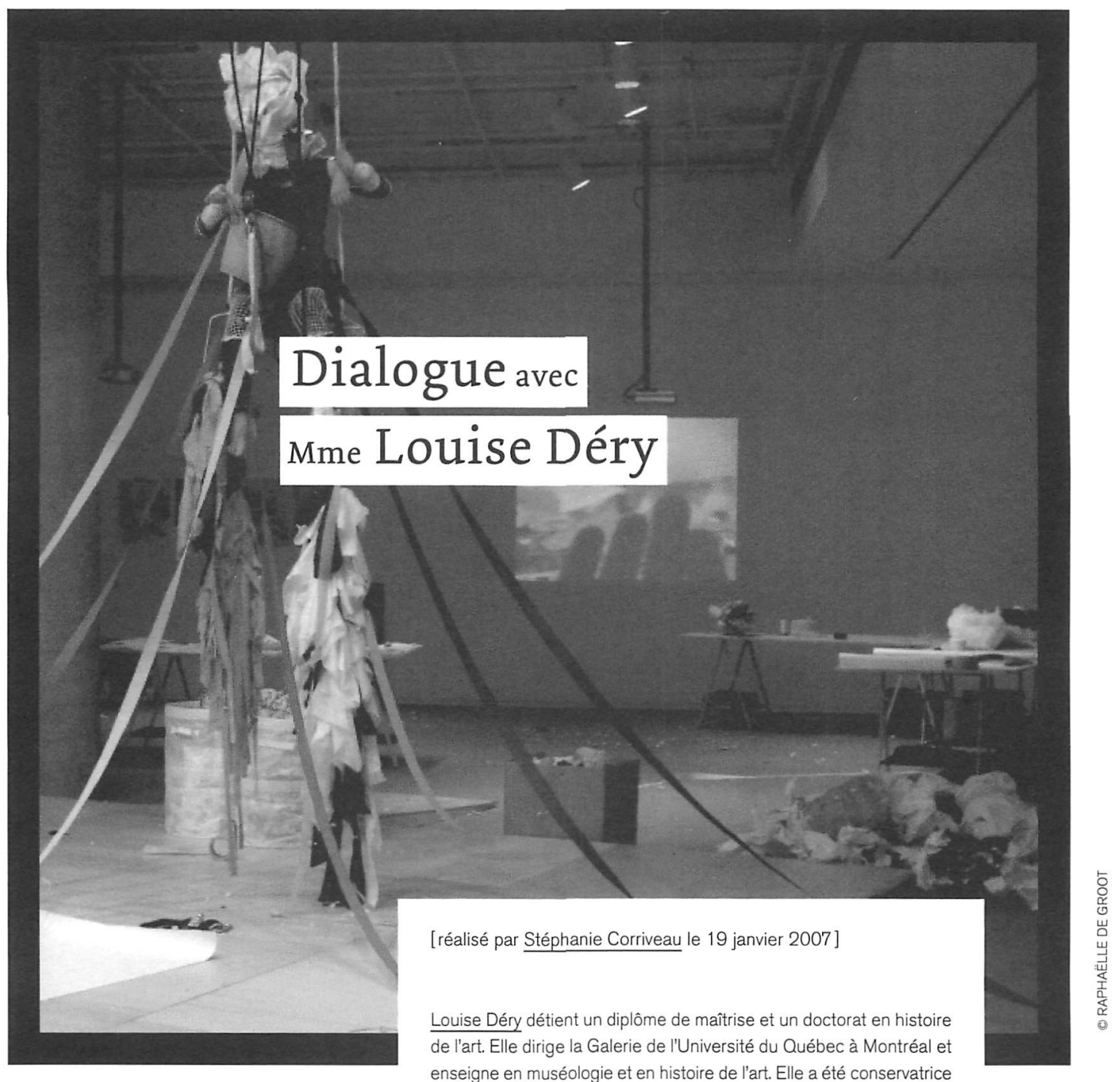
enseigne en muséologie et en histoire de l'art. Elle a été conservatrice au Musée national des beaux-arts du Québec et au Musée des beaux-arts de Montréal. Elle a travaillé entre autres avec Raphaëlle de Groot, Nancy Spero, Giuseppe Penone, Michael Snow, Daniel Buren, Sarkis, Dominique Blain et Jean-Luc Nancy et a publié plus de cinquante catalogues et ouvrages. Commissaire du Canada à la Biennale de Venise 2007, elle y exposera le travail de David Altmejd. La sélection des commissaires canadiens à la Biennale de Venise est organisée par le Conseil des Arts du Canada à l'intérieur d'une compétition nationale ouverte aux commissaires et aux conservateurs d'art contemporain de l'ensemble du Canada. Louise Déry a proposé au jury un projet estimé audacieux, à partir du travail de David Altmejd, de Montréal, qui sera ainsi le plus jeune artiste à représenter le Canada à Venise. Le Canada y a son propre pavillon depuis 1958. En 2005, la Biennale de Venise a reçu 1200000 visiteurs.

CI-DESSUS : l'exposition Raphaëlle de Groot. En exercice présentée à la Galerie de l'UQAM du 24 février au 1ºr avril 2006. Commissaire : Louise Déry 
STÉ Phanie CORRIVEAU

Quels liens faites-vous entre l'histoire de l'art et la muséologie?

LOUISE DÉRY

La plupart des muséologues qui font des expositions d'art sont des historiens de l'art, ce que je suis. Être un historien de l'art, c'est disposer d'un certain savoir, de certaines clés, méthodologiques ou théoriques, pour entrer en contact avec les œuvres d'art. L'historien del'art visite des expositions, mais une bonne partie de sa compréhension provient de la documentation. Quant au muséologue, il ne peut exister sans un rapport direct à l'œuvre. Personnellement, je ne suis pas devenue professeure, mais muséologue; donc j'utilise mes compétences d'historienne de l'art dans les musées plutôt que dans le contexte de l'enseignement universitaire. Le fait de choisir le musée comme véhicule amène une différence d'approche fondamentale. Un commissaire doit mettre de côté son savoir, le garder en veilleuse pour établir un contact ouvert à l'œuvre. Un historien de l'art qui développe une compétence fine de sa spécialité ne sera pas dans un mode expérimental au sens où aime se placer un commissaire d'exposition comme moi. L'exposition peut demeurer sur le plan des idées - elle sera alors idéale, tel que l'affirme Yves Michaud - ou être portée dans la réalité.
STÉPHANIE CORRIVEAU

Comment concevez-vous le commissariat?

LOUISE DÉRY

Je le conçois comme quelque chose de très large. Je pense que c'est le regard que je porte à l'art qui installe dans ma tête des idées de commissariat. C'est la fréquentation de l'art qui fait que l'on développe des préoccupations qui peuvent se transformer en exposition. Que je voie l'œuvre dans un atelier d'artiste, une galerie d'art ou un livre n'a pas d'importance. Ce qui compte c'est la mise au présent qui se fait à partir des œuvres et c'est là que certaines idées s'attachent ensemble ou se rencontrent. La première " pièce de conversation" (conversation piece) d'une exposition s'engage dans la tête du commissaire. J'utilise souvent l'expression : «les œuvres marchent dans ma tête" avant que l'exposition n'existe. Des œuvres, aperçues ou admirées longuement, cherchent parfois à se rencontrer; parfois cette rencontre appelle son lieu et lorsque le lieu est disponible (et c'est le cas quand on travaille dans une institution), cela peut donner une exposition. 
Stéphanie Corriveau

Quel est le statut de l'œuvre d'art dans l'exposition?

\section{LOUISE DÉRY}

L'œuvre d'art a une réalité physique, un poids, des dimensions et une manière d'opérer devant le regard. C'est quelque chose qui est mis dans notre présent au moment où nous en faisons l'expérience. On dit toujours que l'art contemporain est plus une affaire d'expérience que de connaissance. Même si nous ne possédons pas toujours les goûts, les intérêts ou les aptitudes nécessaires pour aborder l'objet, notre premier contact avec lui est toujours physique, ou du moins visuel. L'exposition est l'interface qui nous permet d'aborder l'art de trois manières différentes. Il y a d'abord l'œuvre en elle-même. Elle reflète des moments d'une société, d'une époque et d'une culture qui sont étudiés par l'histoire de l'art et d'autres disciplines, indépendamment $d u$ contexte dans lequel elle est observée. Il y a ensuite "l'œuvre exposée", notion développée parJean-Marc Poinsot dans les années 1980, lorsqu'il s'est aperçu que certaines œuvres, conceptuelles ou de très grandes dimensions, ne peuvent pas être révelées sans qu'elles ne soient exposées. Cela l'a amené à parler des "récits autorisés " et des notions qui permettent d'énoncerl'œuvre dans un lieu. Personnellement, j'aime bien aborder les choses d'une manière différente, simplement en faisant dériver l'expression «l'œuvre exposée» vers "l'œuvre s'exposant ". C'est un concept quej'ai développé au début des années 1990 et qui signifie que, pour moi, exposer l'œuvre, c'est la "déprendre » de son histoire et de son "style " (expression quej'utilise faute de mieux). Nous pouvons voir les œuvres par rapport à l'histoire, à un style, à une thématique, mais le vrai contact que nous avons avec l'œuvre s'établit lorsque celle-ci s'abandonne entièrement à notre regard, lorsqu'elle est "déprise", libérée du discours. Celle-ci ne nous interpelle pas toujours de la même manière, selon les moments de notre vie. Prenons par exemple la lecture : il arrive parfois qu'un livre ne nous intéresse pas nécessairement au premier essai et que nous l'abandonnions. Des années plus tard, nous pouvons nous surprendre à le retrouver et à l'apprécier. C'est la même chose qui arrive avec les œuvres d'art. 
Stéphanie Corriveau

Dans Are You Talking to Me? Conversation(s) ${ }^{[1]}$, vous citez Jean-Hubert Martin qui affirme qu'« une exposition se produit en combinant mentalement des cuvres, en testant leur confrontation et en tentant de les faire dialoguer .. Croyez-vous que les visiteurs soient sensibles au dialogue qu'engendre le côtoiement des couvres?

LOUISE DÉRY

Cette question se pose également pour le commissaire d'expositions - celui-ci étant le premier à tenir le rôle de visiteur-, car les expositions sont une matière à réflexion. Si je vais voir l'œuvre d'un artiste algérien, par exemple, je peux ne pas être très bien outillée pour comprendre la conversation, mais je peux entendre la rumeur. C'est ici que je fais une différence entre un récit ou une pièce de conversation et une rumeur. Une pièce de conversation a une structure. L'intention est explicitée à travers les textes de l'exposition, ce qui permet au visiteur de prendre acte du discours. Il peut $y$ être réceptif, ou pas, comme pour le livre dont il entame la lecture sans qu'elle aboutisse. Par contre, toutes les expositions produisent une rumeur et celleci peut se ressentir comme quelque chose qui nous met à l'aise ou non. Il y a des gens qui, dans une exposition d'art abstrait, ne sont pas

[1]

DÉRY, Louise, Didier PRIOUL et Marie-Pierre SIROIS. Are You Talking to Me? Conversation(s). Montréal : Galerie de l'UOAM, 2003. réceptifs à la rumeur. D'autres sont conscients que des tableaux chromatiques produisent sur eux une expérience sensorielle. La rumeur passe alors par la perception affective de la couleur, de la forme, du sujet, du verbe et de l'espace. Elle peut rejoindre autant le néophyte que le spécialiste, alors que la narration peut très bien ne pas rejoindre le spectateur, surtout s'il n'a pas le désir de voir les œuvres et qu'il ne va pas rechercher quelques aptitudes minimales pour $y$ arriver. La rumeur peut me déplaire lorsque jel'entends, mais il se peut que quinze ans plus tard je me dise : "J'avais pourtant vu ça et je n'ai pas réagi!"

\section{STÉPHANIE CORRIVEAU}

Est-ce qu'il existe, selon vous, des styles d'expositions?

\section{LOUISE DÉRY}

On peut dire que l'exposition est une proposition qui a un style, mais elle n'est pas pour autant une œuvre. On peut dire qu'elle "fait œuvre". La façon dont on a repéré les œuvres et la façon dont on a cherché à les unir donne un style à l'exposition. J'envisage l'exposition comme une mise en scène. La muséographie et la scénographie sont très proches. Lorsque Thomas Bernard est mis en scène par Denis Marleau, cela a le style de Denis Marleau. Or, le texte de Thomas Bernard est ce qui fait œuvre. Le style du metteur en scène va produire la « rumeur» à laquelleje faisais référence plus tôt. Personnellement, je travaille depuis vingt-cinq ans sur une 
certaine plateforme quej'ai êlaborée à partir de mes connaissances et de mes expériences en tant que commissaire. C'est là quej'ai construit ma manière, ma signature. Cependant, chaque fois que je suis en face de productions artistiques nouvelles, cette plateforme n'est plus une zone de sécurité. Il faut que je recommence. J'insiste beaucoup sur le fait que l'on bâtit des aptitudes comme commissaire et que, devant les pratiques artistiques sans cesse nouvelles, on est sans cesse obligé de se méfier de ses compétences et d'en créer d'autres.

STÉPHANIE CORRIVEAU

Faites-vous de l'histoire de l'art à travers les expositions?

LOUISE DÉRY

Je dirais que l'on peut faire de l'histoire de l'art et de "l'histoire de l'art exposée». Faire de l'histoire de l'art exposée, c'est être conscient que l'exposition produit des savoirs et que ces savoirslà, quand on sait les lire à travers l'exposition, on peut en elaborer l'histoire. Certains sont des savoirs surle plan des sciences cognitives.J'ai par exemple en tête l'idée de réaliser une exposition dans laquelle je mettrais ensemble une œuvre de James Turrell portant surla lumière et la couleur avec un tableau de Georges de Latour dans lequel une Madeleine tient une bougie qui produit une lumière et une couleur. C'est une rencontre qui peut produire un savoir sur la lumière. Je pourrais également exposer des portraits d'homme de la peinture française du XVIII' siècle dont on voit les mains. Le fait de les mettre ensemble dans une exposition permettrait de regarder le détail, d'observer comment les peintres à une certaine époque ont traduit la peau. Ce sont des recherches qui sont souvent faites à partir de la documentation et, à mon avis, elles ne sont pas vraiment concluantes tant qu'elles ne sont pas faites de visu. Mettre ensemble les choses dans un espace, les regarder de façon synchronique sont des façons de produire des savoirs. Les thèmes par lesquels nous aménageons des expositions nous obligent à relancer notre vision, parce que, tout à coup, quelqu'un réunit des œuvres que nous n'aurions pas pensévoir ensemble. Par exemple, l'exposition Mémoires d'aveugle réalisée parJacques Derrida au Louvre en 1997 a permis de regrouper les œuvres de nombreux artistes qui ont peu de choses en commun surle plan stylistique, hormis le fait d'avoir exploitéle même thème. Leur rapprochement a produit un nouveau savoir. Il y a aussi des savoirs qui sont purement physiques. Comment agit un monochrome vert de Louis Comtois si je le mets en face d'un paysage chargé d'ormes de Marc-Aurèle Fortin? Il se passe quelque chose sur les plans thématique, chromatique et physique lorsque les œuvres s'abandonnent dans un espace commun. Le savoir est produit par l'expérience que le visiteur fait pendant cette rencontre. Bref, l'histoire de l'art exposée, c'est cette histoire que nous serions amenés à elaborer si nous étions plus attentifs à identifier les savoirs que produisent les expositions. 


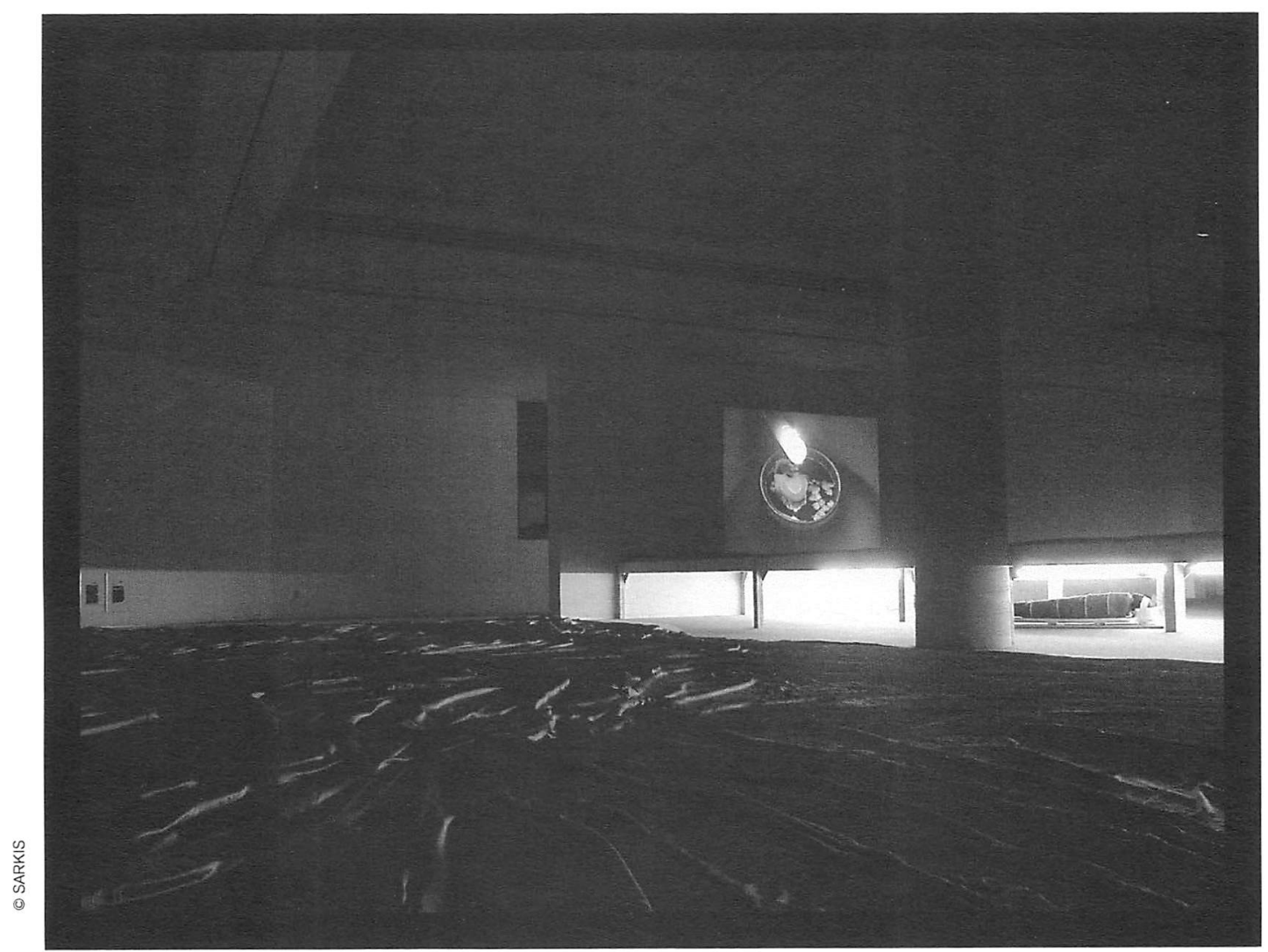

L'exposition Sarkis. 2600 ans après 10 minutes 44 secondes présentée à la Galerie de l'UQAM du 17 octobre au 22 novembre 2003. Commissaire : Louise Déry 
STÉPHANIE CORRIVEAU

Les observateurs de l'art sont-ils, selon vous, sensibles à ces savoirs?

LOUISE DÉRY

Je suis inquiète parle fait que, dansl'histoire et la théorie de l'art, on ne se préoccupe pas beaucoup del'espace d'exposition. On n'en fait pas souvent l'analyse et c'est pour cela que dans mes cours j'insiste beaucoup pour que les étudiants fassent des rapports de visites d'expositions. Qu'est-ce qui se passe dans cette exposition-là, pourquoi $a-t$-on fait les choses comme cela, qu'a-t-on cherché à dire? Les gens se posent très peu ces questions. Ils visitent l'exposition et se concentrent surtout sur l'effet que produit sur eux la découverte d'une œuvre, mais ils considèrent rarement l'expérience del'ensemble et du lieu luimême. Les historiens del'art tardent à développer des théories et des méthodologies d'analyse des expositions. Ils s'attachent à l'œuvre, mais les discours produits parl'exposition sont encore une donnée peu considérée par rapport à cette production de nouveaux savoirs. Au Canada, il n'existe pas une critique de la muséologie très fine. Voilà pourquoi on ne réfléchit guère au fait qu'il peut $y$ avoir de bonnes expositions avec des mauvaises œuvres et de très mauvaises expositions avec des bonnes.
Stéphanie Corriveau

Est-ce que notre manière de faire des expositions est différente au Québec?

LOUISE DÉRY

Nous avons la chance de vivre à une époque qui nous permet de bouger dansl'espace et le temps accompagnés d'une multitude d'outils. Nous n'avons pas, comme le voyageur du XVIIe siècle, à aller voir des œuvres d'art en Italie pour les connaître. Notre regard est constamment sollicité : nous avons la chance de lire Ulysse de James Joyce un jour, puis les textes del'Antiquité le lendemain, et nous pouvons penser que la République de Platon est très actuelle. Alors, à partir du moment où l'on envisage le monde comme un grand réservoir de réalités, il n'y a pas de limites au terrain de jeux que l'on peut se créer. $A u$ Québec, il y a cependant une tendance à se limiter par rapport aux rapprochements qu'il est possible de réaliser entre les œuvres. Lorsqu'on est Américain ou Européen et que l'on possède des collections très anciennes et très variées, les dialogues sont plus faciles à établir. Ici notre désir est le même, mais les limites sont souvent imputables aux questions d'argent ; cela limite les pouvoirs d'imaginer. 
STÉPHANIE CORRIVEAU

Est-ce que le rapprochement entre les ceuvres peut être fait par d'autres véhicules que l'exposition, par exemple les livres?

LOUISE DÉRY

Le livre est un espace d'exposition seulement lorsque l'œuvre lui est destinée par l'artiste. Autrement, les expositions virtuelles sont, enfin pour ma part, de la simple documentation. Je pense avoir été parmi les premiers ici à publier le plan de mes expositions dans les catalogues au début des années 1990. Cela se faisait déjà en Europe, par exemple pour l'exposition Les magiciens de la terre au Centre Pompidou. Je crois que le catalogue d'exposition doit réveler plusieurs choses. Il faut que le rapport à l'espace, qui est l'une des données fondamentales de l'exposition, y soit traduit à l'aide de plans, de graphiques et de photos. La majorité des publications réalisées dans le contexte d'une exposition sont plus souvent des monographies d'artistes que des ouvrages qui traitent des expositions elles-mêmes.

\section{STÉPHANIE CORRIVEAU}

Quels sont les projets de recherche qui vous préoccupent actuellement?

LOUISE DÉRY

J'ai par exemple envisagé avec Didier Prioul, professeur d'histoire de l'art à l'Université Laval, de faire une recherche approfondie sur l'art canadien du $X X^{e}$ siècle, qui permettrait de constater de quelle façon les musées ont accroché leurs collections permanentes. Il existe beaucoup de documentation photographique des accrochages, ce qui faciliterait l'exercice. À la Galerie de l'Université du Quebec à Montréal, par exemple, nous elaborons de la documentation surles expositions, parce que je suis convaincue qu'un jour quelqu'un finira par s'y intéresser. $L a$ vocation d'une galerie universitaire est de donner la possibilité de créer des expositions sur le mode de l'essai, quitte à se tromper, et à les décortiquer afin de voir ce qui fonctionne réellement. Le bonheur serait, selon moi, de tout recommencer, d'essayer de faire existerl'exposition autrement. En ce moment, il y a une exposition à la Galerie : Libre < échange, extraits de la collection de l'UQÀM, pour laquelle j'ai demandé à une jeune commissaire de changer l'emplacement des œuvres en cours de période d'exposition. Le principe est le suivant : on présente un scénario d'exposition, puis on se dit : " on va essayer les choses autrement la semaine prochaine. On va faire sortir cette œuvre-là, puis on va inviter telle autre." On y traite les œuvres comme des personnes qui se rencontrent; elles font un bout de chemin ensemble, puis l'une d'entre elles doit partir, une autre arrive à l'improviste et établit de nouvelles rumeurs. On aurait tort de ne pas se livrer, lorsque cela est possible, à toutes sortes de tentatives afin de réinventer sans cesse les manières d'exposer. Une muséologie vivante, selon moi, part de ce principe. Autrement, il faut prendre sa retraite et laisser la place à d'autres! 